\title{
Inexact Fast Multipole Boundary Element Tearing and Interconnecting Methods
}

\author{
Ulrich Langer ${ }^{12}$, Günther $\mathrm{Of}^{3}$, Olaf Steinbach ${ }^{4}$, and Walter Zulehner ${ }^{1}$ \\ 1 Johannes Kepler University Linz, Institute of Computational Mathematics \\ (http://www.numa.uni-linz.ac.at/) \\ 2 Austrian Academy of Sciences, Johann Radon Institute for Computational and \\ Applied Mathematics, (http://www.ricam.oeaw.ac.at/) \\ 3 University of Stuttgart, Institute for Applied Analysis and Numerical Simulation \\ (http://www.ians.uni-stuttgart/LstAngMath/) \\ 4 TU Graz, Institute of Mathematics, \\ (http://www.numerik.math.tu-graz.ac.at)
}

Summary. The Boundary Element Tearing and Interconnecting (BETI) methods have recently been introduced as boundary element counterparts of the wellestablished Finite Element Tearing and Interconnecting (FETI) methods. In this paper we present inexact data-sparse versions of the BETI methods which avoid the elimination of the primal unknowns and dense matrices. The data-sparse approximation of the matrices and the preconditioners involved is fully based on Fast Multipole Methods (FMM). This leads to robust solvers which are almost optimal with respect to the asymptotic complexity estimates.

\section{Introduction}

Langer and Steinbach [2003] have recently introduced the BETI methods as boundary element counterparts of the well-established FETI methods which were proposed by Farhat and Roux [1991]. We refer the reader to the monograph by Toselli and Widlund [2005] for more information and references to FETI and FETI-DP methods. In particular, we mention the paper by Klawonn and Widlund [2000] who introduced and investigated the inexact FETI technique that avoids the elimination of the primal unknowns (displacements).

In this paper we introduce inexact BETI methods for solving the inhomogeneous Dirichlet boundary value problem (BVP) for the homogeneous potential equation in 3D bounded domains, where all matrices and preconditioners involved in the BETI solver are data-sparse via FMM representations. However, instead of symmetric and positive definite systems, we finally have to solve two-fold saddle point problems. The proposed iterative solver and preconditioner result in an almost optimal solver the complexity of which is proportional to the numbers of unknowns on the skele- 
ton up to some polylogarithmical factor. More precisely, the solver requires $\mathcal{O}\left((H / h)^{(d-1)}(1+\log (H / h))^{4} \log \varepsilon^{-1}\right)$ arithmetical operations in a parallel regime and $\mathcal{O}\left((H / h)^{(d-1)}(1+\log (H / h))^{2}\right)$ storage units per processor, where $d=3$ in the $3 \mathrm{D}$ case considered here, and $\varepsilon \in(0,1)$ is the relative accuracy of the iteration error in a suitable norm. $H$ and $h$ denote the usual scalings of the subdomains and the boundary elements, respectively. Moreover, the solvers are robust with respect to large coefficient jumps. For the sake of simplicity, we present here only the case where all subdomains are non-floating. All results remain valid for the general case that is discussed together with some other issues including other preconditioners in the forthcoming paper by Langer et al. [2005] where the reader can also find the proofs in detail.

The rest of the paper is organized as follows. In Section 2, we introduce the fast multipole boundary element domain decomposition (DD) method. Section 3 is devoted to the inexact BETI method. In Section 4, we describe the ingredients from which the preconditioner and the solver for the two-fold saddle point problem that we finally have to solve is built. In Section 5, we present and discuss the results of our numerical experiments. Finally, we draw some conclusions.

\section{Fast Multipole Boundary Element DD Methods}

Let us consider the Dirichlet BVP for the potential equation

$$
-\operatorname{div}[a(x) \nabla \hat{u}(x)]=0 \text { for } x \in \Omega \subset \mathbb{R}^{3}, \quad \hat{u}(x)=g(x) \text { for } x \in \Gamma=\partial \Omega,
$$

with given Dirichlet data $g \in H^{1 / 2}(\Gamma)$ as a typical model problem, where $\Omega$ is a bounded Lipschitz domain that is assumed to be decomposed into $p$ non-overlapping subdomains $\Omega_{i}$ with Lipschitz boundaries $\Gamma_{i}=\partial \Omega_{i}$. We further assume that the coefficient function $a(\cdot)$ in the potential equation (1) is piecewise constant such that $a(x)=a_{i}>0$ for $x \in \Omega_{i}, i=1, \ldots, p$.

The solution $\hat{u}$ of (1) is obviously harmonic in all subdomains $\Omega_{i}$. Using the representation formula and its normal derivative on $\Gamma_{i}$, we can reformulate the BVP (1) as a DD boundary integral variational problem living on the skeleton $\Gamma_{S}=\cup_{i=1}^{p} \Gamma_{i}$ of the DD, see Costabel [1987] and Hsiao and Wendland [1991]. After homogenization of the Dirichlet boundary condition via the ansatz $\hat{u}=$ $\hat{g}+u$ with $\hat{g}_{\mid \Gamma}=g$ and $u_{\mid \Gamma}=0$, this DD boundary integral variational problem can be written as mixed variational problem of form: find $t=\left(t_{1}, t_{2}, \ldots, t_{p}\right) \in$ $T=T_{1} \times T_{2} \times \ldots \times T_{p}=H^{-1 / 2}\left(\Gamma_{1}\right) \times H^{-1 / 2}\left(\Gamma_{2}\right) \times \ldots \times H^{-1 / 2}\left(\Gamma_{p}\right)$ and $u \in U=\left\{v_{\mid \Gamma_{S}}: v \in H_{0}^{1}(\Omega)\right\}$ such that

$$
a_{i}\left[\left\langle\tau_{i}, V_{i} t_{i}\right\rangle_{\Gamma_{i}}-\left\langle\tau_{i},\left(\frac{1}{2} I+K_{i}\right) u_{\mid \Gamma_{i}}\right\rangle_{\Gamma_{i}}\right]=a_{i}\left\langle\tau_{i},\left(\frac{1}{2} I+K_{i}\right) \hat{g}_{\mid \Gamma_{i}}\right\rangle_{\Gamma_{i}}
$$

for all $\tau_{i} \in T_{i}, i=1,2, \ldots, p$, and 


$$
\sum_{i=1}^{p} a_{i}\left[-\left\langle\left(\frac{1}{2} I+K_{i}^{\prime}\right) t_{i}, v_{\mid \Gamma_{i}}\right\rangle_{\Gamma_{i}}-\left\langle D_{i} u_{\mid \Gamma_{i}}, v_{\mid \Gamma_{i}}\right\rangle_{\Gamma_{i}}\right]=\sum_{i=1}^{p} a_{i}\left\langle D_{i} \hat{g}_{\mid \Gamma_{i}}, v_{\mid \Gamma_{i}}\right\rangle_{\Gamma_{i}}
$$

for all $v \in U$, where $V_{i}, K_{i}, K_{i}^{\prime}$, and $D_{i}$ denote the local single layer potential operator, the local double layer potential operator, its adjoint, and the local hypersingular boundary integral operator, respectively.

Let us now introduce the boundary element trial spaces $U_{h}=S_{h}^{1}\left(\Gamma_{S}\right)=$ $\operatorname{span}\left\{\varphi_{m}\right\}_{m=1}^{M} \subset U$ and $T_{i, h}=S_{h}^{0}\left(\Gamma_{i}\right)=\operatorname{span}\left\{\psi_{k}^{i}\right\}_{k=1}^{N_{i}} \subset T_{i}$ spanned by continuous piecewise linear basis functions $\varphi_{m}$ and by piecewise constant basis functions $\psi_{k}^{i}$ with respect to a regular globally quasi-uniform boundary element mesh with the average mesh size $h$ on $\Gamma_{S}$ and $\Gamma_{i}$, respectively. The Galerkin discretization finally leads to a large-scale symmetric and indefinite system of form

$$
\left(\begin{array}{cccc}
a_{1} \widetilde{V}_{1, h} & & & -a_{1} \widetilde{K}_{1, h} R_{1, h} \\
& \ddots & & \vdots \\
& & a_{p} \widetilde{V}_{p, h} & -a_{p} \widetilde{K}_{p, h} R_{p, h} \\
-a_{1} R_{1, h}^{\top} \widetilde{K}_{1, h}^{\top} & \ldots & -a_{p} R_{p, h}^{\top} \widetilde{K}_{p, h}^{\top} & -\widetilde{D}_{h}
\end{array}\right)\left(\begin{array}{c}
\widetilde{\underline{t}}_{1} \\
\vdots \\
\underline{\widetilde{t}}_{p} \\
\underline{\widetilde{u}}
\end{array}\right)=\left(\begin{array}{c}
a_{1} \underline{\widetilde{g}}_{1} \\
\vdots \\
a_{p} \underline{\widetilde{g}} p \\
\underline{\widetilde{f}}
\end{array}\right)
$$

for defining the coefficient vectors $\widetilde{\underline{t}}_{i} \in \mathbb{R}^{N_{i}}$ and $\underline{\widetilde{u}} \in \mathbb{R}^{M}$. The matrices $\widetilde{V}_{i, h}$, $\widetilde{K}_{i, h}$ and $\widetilde{D}_{h}$ are data-sparse FMM approximations to the originally dense Galerkin matrices $V_{i, h}, K_{i, h}$ and $D_{h}=\sum_{i=1}^{p} a_{i} R_{i, h}^{\top} D_{i, h} R_{i, h}$, respectively. The use of the FMM is indicated by the "tilde" on the matrices and vectors. The FMM approximation of these matrices reduces the quadratic complexity with respect to the number of unknowns to an almost linear one, but without disturbing the accuracy. The restriction operator $R_{i, h}$ maps some global coefficient vector $\underline{v} \in \mathbb{R}^{M}$ to the local vector $\underline{v}_{i} \in \mathbb{R}^{M_{i}}$ containing those components of $\underline{v}$ which correspond to $\Gamma_{i}$ only, $i=1,2, \ldots, p$. The matrices $R_{i, h}$ are Boolean matrices which are sometimes also called subdomain connectivity matrices.

\section{Inexact BETI Methods}

Introducing the local unknowns $\underline{\widetilde{u}}_{i}=R_{i, h} \underline{\widetilde{u}}$ as individual variables and enforcing again the global continuity of the potentials by the constraints

$$
\sum_{i=1}^{p} B_{i} \tilde{\underline{u}}_{i}=\underline{0}
$$

we immediately arrive at the two-fold saddle point problem

$$
\left(\begin{array}{ccc}
V & K & 0 \\
K^{\top} & -D & B^{\top} \\
0 & B & 0
\end{array}\right)\left(\begin{array}{l}
\underline{t} \\
\underline{u} \\
\underline{\lambda}
\end{array}\right)=\left(\begin{array}{l}
\underline{g} \\
\underline{f} \\
\underline{0}
\end{array}\right)
$$


that is obviously equivalent to $(4)$, where $\underline{t}=\left(\underline{\widetilde{t}}_{1}, \ldots, \underline{\underline{t}}_{p}\right)^{\top}, \underline{u}=\left(\underline{\widetilde{u}}_{1}, \ldots, \underline{\widetilde{u}}_{p}\right)^{\top}$, and $\underline{\lambda} \in \mathbb{R}^{L}$ is the vector of the Lagrange multipliers. The matrices $V=$ $\operatorname{diag}\left(a_{i} \widetilde{V}_{i, h}\right), K=\operatorname{diag}\left(-a_{i} \widetilde{K}_{i, h}\right)$ and $D=\operatorname{diag}\left(a_{i} \widetilde{D}_{i, h}\right)$ are block-diagonal whereas $B=\left(B_{1}, \ldots, B_{p}\right)$. As in the FETI method each row of the matrix $B$ is connected with a pair of matching nodes across the subdomain boundaries. The entries of such a row are 1 and -1 for the indices corresponding to the matching nodes on the interface (coupling boundaries) $\Gamma_{C}=\Gamma_{S} \backslash \Gamma$ and 0 otherwise. We assume here that the number of constraints at some matching node is equal to the number of matching subdomains minus one. This method of a minimal number of constraints respectively multipliers is called non-redundant (see, e.g., Toselli and Widlund [2005]). The matrices $\widetilde{V}_{i, h}$ are symmetric and positive definite (SPD). For non-floating subdomains assumed in this paper the matrices $\widetilde{D}_{i, h}$ are SPD as well. In the more complicated case of floating subdomains, the matrices $\widetilde{D}_{i, h}$ must be modified due to the nontrivial kernel $\operatorname{ker}\left(\widetilde{D}_{i, h}\right)=\operatorname{span}\left\{\underline{1}_{i}\right\}$, where $\left\{\underline{1}_{i}\right\}=(1, \ldots, 1)^{\top}$, see Langer and Steinbach [2003] or Langer et al. [2005].

\section{Solvers and Preconditioners}

Following Zulehner [2005], who extended the special conjugate gradient (CG) method proposed by Bramble and Pasciak [1988] for solving one-fold saddle point problems, to $\mathrm{n}$-fold saddle point problems, we are able to construct a very efficient saddle point conjugate gradient (SPCG) solver for our twofold saddle point problem (6) provided that appropriate precondtioners for the single layer potential matrices $\widetilde{V}_{i, h}$, the local boundary element Schur complements $\widetilde{S}_{i, h}=\widetilde{D}_{i, h}+\widetilde{K}_{i, h}^{\top} \widetilde{V}_{i, h}^{-1} \widetilde{K}_{i, h}$ and the BETI Schur complement $\widetilde{F}=\sum_{i=q+1}^{p} a_{i}^{-1} B_{i} \widetilde{S}_{i, h}^{-1} B_{i}^{\top}$ are available. We propose the following datasparse preconditioners which are also used in our numerical experiments:

1. Data-sparse algebraic or geometric multigrid preconditoners $\widetilde{\mathcal{V}}_{i, h}$ for the matrices $\widetilde{V}_{i, h}$ : For the geometric multigrid method, Langer and Pusch [2005] proved the spectral equivalence inequalities

$$
\underline{c}_{V} \widetilde{\mathcal{V}}_{i, h} \leq \widetilde{V}_{i, h} \leq \bar{c}_{V} \widetilde{\mathcal{V}}_{i, h}
$$

where the spectral equivalence constants $\underline{c}_{V}$ and $\bar{c}_{V}$ are positive and independent of $h$ and $H$.

2. Data-sparse opposite order preconditioners $\widetilde{\mathcal{S}}_{i, h}$ for the local boundary element Schur complements $\widetilde{S}_{i, h}$ : In order to construct efficient preconditioners $\widetilde{\mathcal{S}}_{i, h}$, we apply the concept of boundary integral operators of the opposite order proposed by Steinbach and Wendland [1998]. Based on the local trial space $U_{i, h}=S_{h}^{1}\left(\Gamma_{i}\right)$ of piecewise linear basis functions $\varphi_{m}^{i}$, as used for the Galerkin discretization of the local hypersingular boundary integral operators $D_{i}$, we define the Galerkin matrices $\bar{V}_{i, h}$ and $\bar{M}_{i, h}$ by 


$$
\bar{V}_{i, h}[n, m]=\left\langle\varphi_{n}^{i}, V \varphi_{m}^{i}\right\rangle_{\Gamma_{i}}, \quad \bar{M}_{i, h}[n, m]=\left\langle\varphi_{n}^{i}, \varphi_{m}^{i}\right\rangle_{\Gamma_{i}}
$$

for $m, n=1, \ldots, M_{i}$. The inverse preconditioners are now defined by

$$
\widetilde{\mathcal{S}}_{i, h}^{-1}=\bar{M}_{i, h}^{-1} \widetilde{\widetilde{V}}_{i, h} \bar{M}_{i, h}^{-1} \quad \text { for } i=1, \ldots, p,
$$

where the tilde on the top of $\widetilde{\bar{V}}_{i, h}$ again indicates that the application of the discrete single layer potential $\bar{V}_{i, h}$ is realized by using the FMM. In Langer et al. [2005] we prove the spectral equivalence inequalities

$$
\underline{c}_{S}(1+\log (H / h))^{-2} \widetilde{\mathcal{S}}_{i, h} \leq \widetilde{S}_{i, h} \leq \bar{c}_{S} \widetilde{\mathcal{S}}_{i, h},
$$

where the spectral equivalence constants $\underline{c}_{S}$ and $\bar{c}_{S}$ are positive and independent of $h$ and $H$. The log-term disappears in the case of floating subdomains.

3. Data-sparse BETI preconditioner $\widetilde{\mathcal{F}}$ for the BETI Schur complements $\widetilde{F}$ : Following Langer and Steinbach [2003], we define the inverse BETI preconditioner

$$
\widetilde{\mathcal{F}}_{i, h}^{-1}=\left(B C_{a}^{-1} B^{T}\right)^{-1} \sum_{i=1}^{p} B_{i} C_{\alpha}^{-1} \widetilde{D}_{i, h} C_{a, i}^{-1} B_{i}^{\top}\left(B C_{a}^{-1} B^{\top}\right)^{-1} .
$$

with the help of the local data-sparse discrete hypersingular operators $\widetilde{D}_{i, h}$ and the scaling matrix $C_{a}=\operatorname{diag}\left(C_{a, i}\right)$. The definition of the diagonal matrices $C_{a, i}$ can be found in Toselli and Widlund [2005]. In Langer et al. [2005], the spectral equivalence inequalities

$$
\underline{c}_{F} \widetilde{\mathcal{F}} \leq \widetilde{F} \leq \bar{c}_{F}(1+\log (H / h))^{2} \widetilde{\mathcal{F}}
$$

were proved, where the spectral equivalence constants $\underline{c}_{S}$ and $\bar{c}_{S}$ are positive and independent of $h, H$ and the $a_{i}$ 's (coefficients jumps). In the general case where non-floating as well as floating subdomains are present in the DD, the spectral equivalence inequalities (11) remain valid on an appropriate subspace.

Combining these spectral equivalence estimates with the results obtained by Zulehner [2005] and taking into account the complexity estimate for the FMM, we can easily prove the following theorem.

Theorem 1. If the two-fold saddle point problem (6) is solved by the SPCG method where the preconditioner is build from the block preconditioners $\widetilde{\mathcal{V}}_{i, h}$, $\widetilde{\mathcal{S}}_{i, h}$, and $\widetilde{\mathcal{F}}$, then not more than $I(\varepsilon)=\mathcal{O}\left((1+\log (H / h))^{2} \log \varepsilon^{-1}\right)$ iterations and ops $(\varepsilon)=\mathcal{O}\left((H / h)^{2}(1+\log (H / h))^{4} \log \varepsilon^{-1}\right)$ arithmetical operations are required in order to reduce the initial error by the factor $\varepsilon \in(0,1)$ in a parallel regime. The number of iterations $I(\varepsilon)$ is robust with respect to the jumps in the coefficients. Moreover, not more than $\mathcal{O}\left((H / h)^{2}(1+\log (H / h))^{2}\right)$ storage units are needed per processor. 
The results of the theorem remain valid also in the general case where also floating subdomains are present in the domain decomposition (see Langer et al. [2005]). The proposed SPCG solver is asymptotically almost optimal with respect to the complexity in arithmetic and storage as well as very efficient on a parallel computer with distributed memory.

Remark 1. If we would use optimal preconditioners $\widetilde{\mathcal{S}}_{i, h}$ for the local boundary element Schur complements $\widetilde{S}_{i, h}$, then the number of iteration $I(\varepsilon)$ of our SPCG solver would behave like $\mathcal{O}\left((1+\log (H / h)) \log \varepsilon^{-1}\right)$, whereas the arithmetical complexity would decrease from $\mathcal{O}\left((H / h)^{2}(1+\log (H / h))^{4} \log \varepsilon^{-1}\right)$ to $\mathcal{O}\left((H / h)^{2}(1+\log (H / h))^{3} \log \varepsilon^{-1}\right)$. Such preconditioners are available. If we convert the non-floating subdomains having a Dirichlet boundary part to floating subdomains by including the Dirichlet boundary condition into the constraints, then the data-sparse opposite order preconditioners $\widetilde{\mathcal{S}}_{i, h}$ given above is optimal.

\section{Numerical Results}

Let us consider the unit cube which is subdivided into eight similar subdomains. In order to check the behavior of the discretization error, we take the Dirichlet data $g=\hat{u}_{\mid \Gamma}$ as the trace of a regular solution $\hat{u}$ of the boundary value problem (1) on the boundary $\Gamma$. We perform numerical experiments for the Laplace equation $\left(a_{i}=1\right.$ for all $\left.i=1, \ldots, 8\right)$ and for the potential equation with large jumps in the coefficients $\left(a_{i} \in\left\{1,10^{5}\right\}\right)$ (chequerboard distribution).

Starting from the coarsest grid level $L=0$ with 192 triangles on $\cup \partial \Omega_{i}$, we successively refine the mesh by subdividing each triangle into four smaller similar triangles. $N$ and $M$ denote the total numbers of triangles and nodes, respectively. $M_{c}$ is the total number of coupling nodes. The numbers of local triangles and nodes on $\partial \Omega_{i}$ are given by $N_{i}$ and $M_{i}$, respectively. If the boundary mesh of one subdomain $\Omega_{i}$ on level $L=6$ with 98.304 triangles is uniformly extended to the interior of the subdomain, then the corresponding finite element mesh would consist of 4.448 .731 tetrahedrals resulting in almost 36 millions tetrahedrals for the whole computational domain. In Table 1, together with the mesh features $L, N, M, M_{c}, N_{i}$ and $M_{i}$, the time $t_{1}$ [sec] for generating the system (6) and for setting up the preconditioner, the time $t_{2}$ [sec] spent by the SPCG solver, the number of iterations $I(\varepsilon)$ and the absolute $L_{2}\left(\Gamma_{i}\right)$ discretization error $\left\|\hat{u}-\hat{u}_{h}\right\|_{L_{2}\left(\Gamma_{i}\right)}$ are displayed. The relative accuracy $\varepsilon$ of the iteration error is chosen to be $10^{-8}$. The first line in each row for the columns $t_{1}, t_{2}, I(\varepsilon)$ and $L_{2}\left(\Gamma_{i}\right)$-error corresponds to the Laplace case whereas the second line corresponds to the case of jumping coefficients. Table 1 shows that the growth in the number of iterations and in the CPU times is in good agreement with the complexity estimates given in Theorem 1 . The efficiency of our SPCG solver is not affected by large jumps in the coefficients of the 


\begin{tabular}{r|r|r|r|r|r|r|r|r|r}
\hline \hline$L$ & $N$ & $M$ & $M_{c}$ & $N_{i}$ & $M_{i}$ & $t_{1}$ & $t_{2}$ & $I(\varepsilon)$ & $L_{2}$-error \\
\hline 0 & 192 & 63 & 13 & 24 & 14 & 0 & 0 & 6 & $2,8527 \mathrm{E}-03$ \\
& & & & & & 1 & 0 & 6 & $2,8527 \mathrm{E}-08$ \\
\hline 1 & 768 & 261 & 67 & 96 & 50 & 1 & 1 & 33 & $7,1318 \mathrm{E}-04$ \\
& & & & & & 1 & 1 & 29 & $7,1318 \mathrm{E}-09$ \\
\hline 2 & 3072 & 1089 & 319 & 384 & 194 & 5 & 6 & 36 & $1,7830 \mathrm{E}-04$ \\
& & & & & & 5 & 6 & 34 & $1,7830 \mathrm{E}-09$ \\
\hline 3 & 12288 & 4473 & 1399 & 1536 & 770 & 16 & 34 & 38 & $4,4574 \mathrm{E}-05$ \\
& & & & & & 15 & 30 & 36 & $4,4577 \mathrm{E}-10$ \\
\hline 4 & 49152 & 18153 & 5863 & 6144 & 3074 & 81 & 186 & 41 & $1,1143 \mathrm{E}-05$ \\
& & & & & & 79 & 172 & 38 & $1,1144 \mathrm{E}-10$ \\
\hline 5 & 196608 & 73161 & 24007 & 24576 & 12290 & 316 & 1469 & 46 & $2,7859 \mathrm{E}-06$ \\
& & & & & & 310 & 1346 & 44 & $2,7859 \mathrm{E}-11$ \\
\hline 6 & 786432 & 293769 & 97159 & 98304 & 49154 & 1314 & 7250 & 55 & $6,9647 \mathrm{E}-07$ \\
& & & & & & 1319 & 7034 & 49 & $6,9651 \mathrm{E}-12$ \\
\hline \hline
\end{tabular}

Table 1. Numerical features for the SPCG solver.

potential equations (1). Moreover, the number of iterations are less than in the Laplace case. In addition, the CPU time for the finest level $L=6$ is half of the time needed for a primal preconditioned Schur complement solver in the case of jumping coefficients. All numerical experiments were performed on standard PCs with 3.06 Mhz Intel processors and 1 GB of RAM.

\section{Conclusions}

Inexact data-sparse BETI methods introduced in this paper show an almost optimal behavior with respect to the number of iterations, the arithmetical costs and the memory consumption. Moreover, the methods are robust with respect to large jumps in the coefficients of (1). These results were rigorously proved and were also confirmed by our numerical experiments. The treatment of the outer Dirichlet problem as well as other boundary conditions is straightforward. Inexact data-sparse BETI methods can naturally be generalized to linear elasticity BVP including elasticity problems for almost incompressible materials (cf. Steinbach [2003]). Combining the results of this paper with the results on inexact FETI methods obtained by Klawonn and Widlund [2000], we can develop inexact BETI-FETI solvers for coupled boundary and finite element equations (cf. Langer and Steinbach [2004] for the exact version).

Acknowledgement. This work has been supported by the Austrian Science Fund 'Fonds zur Förderung der wissenschaftlichen Forschung (FWF)' under the grants P14953 and SFB F013 'Numerical and Symbolic Scientific Computing', and by the German Research Foundation 'Deutsche Forschungsgemeinschaft (DFG)' under the grant SFB 404 'Multifield Problems in Continuum Mechanics'. 


\section{References}

J. H. Bramble and J. E. Pasciak. A preconditioning technique for indefinite systems resulting from mixed approximations of elliptic problems. Math. Comp., 50(181):1-17, 1988.

M. Costabel. Symmetric methods for the coupling of finite elements and boundary elements. In C. A. Brebbia, W. L. Wendland, and G. Kuhn, editors, Boundary Elements IX, pages 411-420, Berlin, Heidelberg, New York, 1987. Springer.

C. Farhat and F.-X. Roux. A method of finite element tearing and interconnecting and its parallel solution algorithm. Int. J. Numer. Meth. Engrg., 32:1205-1227, 1991.

G. C. Hsiao and W. L. Wendland. Domain decomposition in boundary element methods. In Proceedings of the Fourth International Symposium on Domain Decomposition Methods for Partial Differential Equations (ed. by R. Glowinski and Y. A. Kuznetsov and G. Meurant and J. Périaux and $O$. B. Widlund), Moscow, May 21-25, 1990, pages 41-49, Philadelphia, 1991. SIAM.

A. Klawonn and O. B. Widlund. A domain decomposition method with Lagrange multipliers and inexact solvers for linear elasticity. SIAM J. Sci. Comput., 22(4):1199-1219, 2000.

U. Langer, G. Of, O. Steinbach, and W. Zulehner. Inexact data-sparse boundary element tearing and interconnecting methods. Technical Report, Johann Radon Institute for Computational and Applied Mathematics, Autrian Academy of Sciences, Linz, Austria, 2005.

U. Langer and D. Pusch. Convergence analysis of geometrical multigrid methods for solving data-sparse boundary element equations. SFB-Report, SFB F013, Johannes Kepler University Linz, Austria, 2005.

U. Langer and O. Steinbach. Boundary element tearing and interconnecting method. Computing, 71:205-228, 2003.

U. Langer and O. Steinbach. Coupled boundary and finite element tearing and interconnecting methods. In Proceedings of the 15th Int. Conference on Domain Decomposition (ed. by R. Kornhuber, R. Hoppe, J. Periaux, O. Pironneau, O. Widlund and J. Xu), Lecture Notes in Computational Sciences and Engineering, vol. 40, pages 83-97, Heidelberg, 2004. Springer.

O. Steinbach. A robust boundary element method for nearly incompressible linear elasticity. Numer. Math., 95:553-562, 2003.

O. Steinbach and W. L. Wendland. The construction of some efficient preconditioners in the boundary element method. Adv. Comput. Math., 9:191-216, 1998.

A. Toselli and O. Widlund. Domain Decomposition Methods - Algorithms and Theory, volume 34 of Springer Series in Computational Mathematics. Springer, Berlin, Heidelberg, 2005.

W. Zulehner. Uzawa-type methods for block-structured indefinite linear systems. SFB-Report 2005-5, SFB F013, University of Linz, Austria, 2005. 
